\title{
Iron-Catalyzed Radical Intermolecular Addition of Unbiased Alkenes to Aldehydes
}

\author{
Mar Saladrigas, Jordi Puig, Josep Bonjoch,* and Ben Bradshaw* \\ Laboratori de Química Orgànica, Facultat de Farmacia, IBUB, Universitat de Barcelona, Av. Joan XXIII s/n, 08028- \\ Barcelona, Spain.
}

\begin{abstract}
The intermolecular reductive radical coupling of aldehydes with non-activated alkenes, employing metal hydride atom transfer (MHAT) catalysis with a combination of $\mathrm{Fe}^{\mathrm{II}}$ and $\mathrm{Fe}^{\mathrm{III}}$ salts, is described. This constitutes the first use of aldehydes as viable acceptor groups in MHAT reactions. The insights gained in this study led to the reexamination of the previously reported intramolecular version of the reaction, and the addition of $\mathrm{Fe}^{\mathrm{II}}$ salts allowed the development of a more efficient second-generation approach.
\end{abstract}

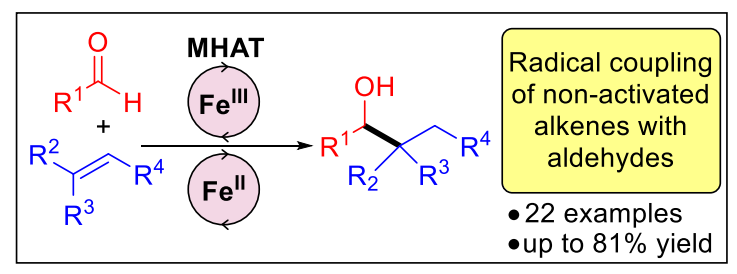

A long-standing challenge in the field of radical chemistry is the use of $\mathrm{C}=\mathrm{O}$ bonds (aldehydes or ketones), one of the most common functionalities in organic chemistry, as acceptor groups. Despite the feasibility of radical addition, the thermodynamic instability of the resulting alkoxyl radical ${ }^{1}$ rapidly leads to homolytic cleavage of the coupled product, which reverts back to the more stable initial carbon-centered radical via $\beta$-fragmentation. ${ }^{2}$ Indeed, the proclivity to this reverse reaction is such that it is often used to cleave alcohols across adjacent $\mathrm{C}-\mathrm{C}$ bonds to generate carbonyl compounds $^{3}$ (Figure 1A).

Various strategies to overcome this energetically unfavourable addition have been devised, as outlined in Figure $1 \mathrm{~B}$. One of the most successful approaches is to tether the carbonyl to the radical precursor, ${ }^{4}$ which we illustrated when demonstrating that ketone ${ }^{5}$ can serve as viable radical acceptors under metal hydride atom transfer (MHAT) ${ }^{6}$ conditions (Scheme 1Bi). ${ }^{7}$ However, our initial attempts to carry out the intermolecular variant of this reaction were unsuccessful. Moreover, very few examples of intermolecular radical coupling have been reported in the literature. Glorius was able to carry out the intermolecular radical addition to aldehydes by using visible-light photoredox initiated hole catalysis in combination with in situ Brønsted acid activation of the carbonyl (Figure 1 Bii). ${ }^{8}$ This resulted in a favourable thermodynamic driving force while also kinetically improving the rate of the electron transfer step. Finally, a third possible approach involves transmetalation of the initially formed radical, which circumvents the formation of the unfavourable alkoxyl radical species altogether. For example, the Shenvi group developed a radical polar crossover strategy using a MHAT reaction to form a putative organocobalt species, which then was transmetalated with chromium to allow a subsequent Nozaki-Hiyama type coupling reaction (Scheme 1Biii). ${ }^{9}$ Interestingly, as we had previously observed, their attempts to carry out the
MHAT coupling without the transmetalation step provided only trace amounts of the coupled product.

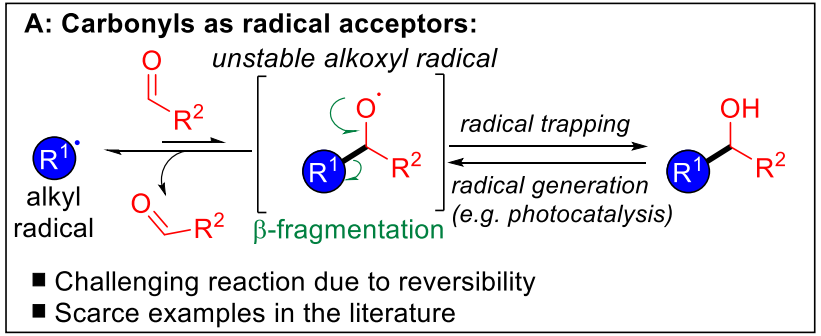

B: Strategies used to overcome reaction reversibility

(i) Bradshaw \& Bonjoch: Intramolecular MHAT coupling of ketones

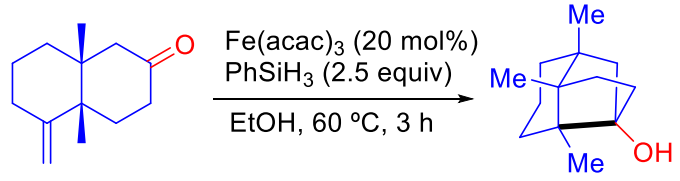

(ii) Glorius: Photocatalytic "hole" catalysis

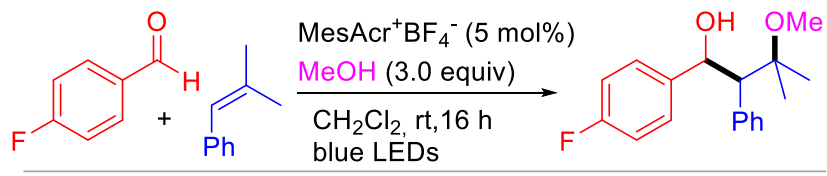

(iii) Shenvi: HAT to Nozaki-Hiyama coupling via radical crossover

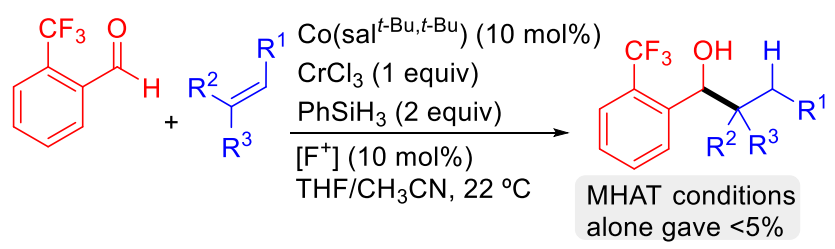

Figure 1. Using carbonyls as radical acceptors. 
After our subsequent experience in developing a successful MHAT intermolecular coupling of $\mathrm{Cbz}$ hydrazones to access amines ${ }^{10}$ as well as tosylhydrazones ${ }^{11}$ as a general alkylation reaction, we decided to revisit the intermolecular MHAT coupling reaction of aldehydes. Based on both experimental observations and mechanistic considerations, ${ }^{12}$ we proposed a new strategy involving the addition of $\mathrm{Fe}^{\mathrm{II}}$, which could play multiple positive roles within the catalytic cycle of the reaction (Figure 2). It was envisaged that the addition of $\mathrm{Fe}^{\mathrm{II}}$ would facilitate the SET process, enabling a faster reduction and rapid entrapment of the formed alkoxyl radical, and thus pre-empt a reverse reaction via $\beta$ fragmentation (option A). Secondly, by reacting with the initially formed carbon radical species (option B), $\mathrm{Fe}^{\mathrm{II}}$ would stabilize the radical via the persistent radical effect (P.R.E) ${ }^{12 b, 13}$ and prevent its loss before the desired reaction could take place. Finally, Fe ${ }^{\mathrm{II}}$ may act as a Lewis acid, lowering the activation energy of the reaction ${ }^{8}$ and subsequently facilitating a direct SET process (option C). We report here the validation of this theory and the first successful use of aldehydes as radical acceptors in MHAT reactions.

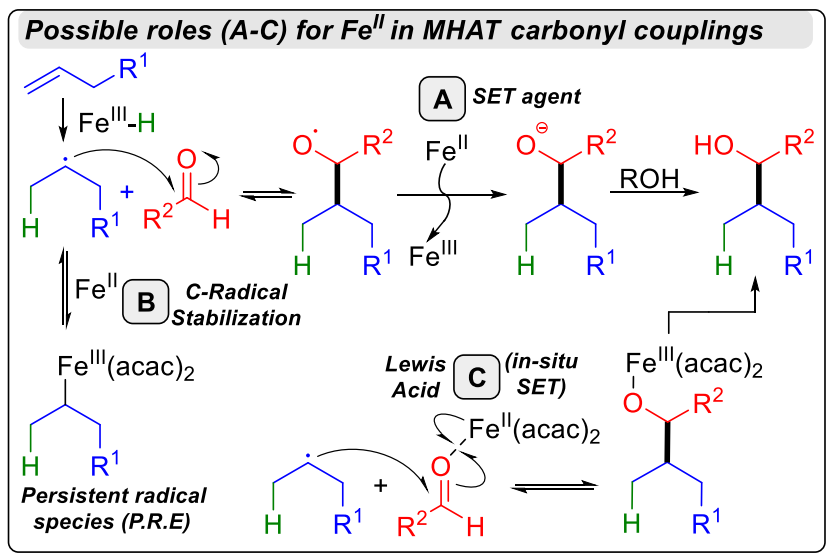

Figure 2. Possible beneficial roles of FeII in the MHAT coupling reaction of non-activated alkenes with aldehydes.

To evaluate the reaction parameters, based on our intermolecular MHAT couplings of alkenes with hydrazones, ${ }^{10} 4$ cyanobenzaldehyde 1a was chosen as the acceptor group and 4-phenyl-1-butene $\mathbf{2 a}$ as the radical precursor. As can be seen, the use of $\mathrm{Fe}^{\mathrm{III}}$ alone in $\mathrm{EtOH}$ gave the coupled product $3 \mathbf{a}$ in very low yields (7\%) (Table 1, entry 1), which were moderately improved using stoichiometric quantities of $\mathrm{Fe}(\mathrm{acac})_{3}$ in EtOH (entry 2). As in all our previously developed intermolecular MHAT coupling reactions, ${ }^{9,10}$ heating was found to be detrimental (entry 3). We then began to evaluate the effect of adding $\mathrm{Fe}^{\mathrm{II}}$ to the reaction, observing a slight increase in yield to $32 \%$ when using stoichiometric iron in a $2: 8 \mathrm{Fe}^{\mathrm{III}} / \mathrm{Fe}^{\mathrm{II}}$ ratio (entry 4 ), although the improvement was far less than expected.

However, after changing the solvent from EtOH to THF with $\mathrm{MeOH}$ as an additive (2 equiv), ${ }^{14}$ a synthetically useful yield (48\%) was obtained for the first time (entry 5). Changing the Fe ratio to 1:1 led to a minor improvement (entry 6). In further tests, increasing the amount of $\mathrm{MeOH}$ to 10 equiv resulted in a higher yield (entry 7), but 20 equiv led to only a slight improvement compared to 2 equiv.
Table 1. Screening of reaction conditions

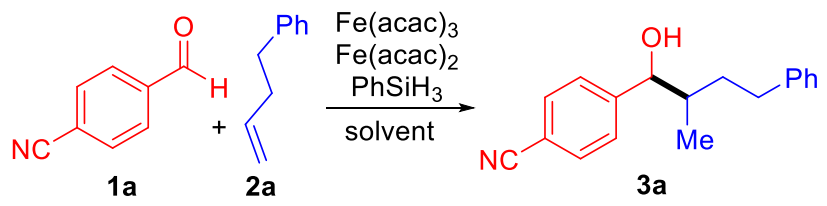

$\begin{array}{ccccccc}\text { entry } & \mathbf{1 a} & \mathbf{2 a} & \text { Fe }^{\text {III }} / \mathbf{F e}^{I I} & \text { solvent } & \text { time } & \text { yield } \\ \mathbf{1} & 1 & 1 & 0.2: 0 & \mathrm{EtOH} & 24 \mathrm{~h} & 7 \% \\ \mathbf{2} & 1 & 1 & 1: 0 & \mathrm{EtOH} & 24 \mathrm{~h} & 25 \% \\ \mathbf{3}^{\mathrm{a}} & 1 & 1 & 1: 0 & \mathrm{EtOH} & 24 \mathrm{~h} & 10 \% \\ \mathbf{4} & 1 & 1 & 0.2: 0.8 & \mathrm{EtOH} & 24 \mathrm{~h} & 32 \% \\ \mathbf{5} & 1 & 1 & 0.2: 0.8 & \mathrm{THFb} & 24 \mathrm{~h} & 48 \% \\ \mathbf{6} & 1 & 1 & 0.5: 0.5 & \mathrm{THFb} & 24 \mathrm{~h} & 50 \% \\ \mathbf{7} & 1 & 1 & 0.5: 0.5 & \mathrm{THFc} & 24 \mathrm{~h} & 70 \% \\ \mathbf{8} & 1 & 1 & 0: 1 & \mathrm{THFc} & 24 \mathrm{~h} & 35 \% \\ \mathbf{9} & 2 & 1 & 0.5: 0.5 & \mathrm{THFc} & 24 \mathrm{~h} & 69 \% \\ \mathbf{1 0} & 1 & 2 & 0.5: 0.5 & \mathrm{THF} & 24 \mathrm{~h} & 58 \% \\ \mathbf{1 1} & 1 & 1 & 0.5: 0.5 & \mathrm{THFc} & 8 \mathrm{~h} & 69 \% \\ \mathbf{1 2} & 1 & 1 & 0.2: 0.2 & \mathrm{THFc} & 8 \mathrm{~h} & 59 \%\end{array}$

${ }^{a}$ Heated at $60{ }^{\circ} \mathrm{C} .{ }^{b} 2$ equiv of $\mathrm{MeOH}$ as additive. ${ }^{c} 10$ equiv of $\mathrm{MeOH}$ as additive. ${ }^{d}$ Open to air.

The use of $\mathrm{Fe}^{\mathrm{II}}$ alone, open to the air, gave a lower yield (entry 8), probably due to competing Mukaiyama oxidation. ${ }^{15}$ Next, when evaluating the acceptor $\mathbf{1 a}$ /donor $\mathbf{2 a}$ ratio, the yield was found to be unaffected by increasing the amounts of 1a (entry 9). Unexpectedly, however, adding more alkene $\mathbf{2 a}$ proved detrimental, likely because the alkene is the least stable component in the reaction mixture and more prone to side reactions before the coupling takes place (entry 10). Cutting the reaction time from $24 \mathrm{~h}$ to $8 \mathrm{~h}$ produced almost identical results (entry 11), whereas a further reduction to $3 \mathrm{~h}$ proved unfeasible. Reducing the quantity of Fe to substoichiometric amounts $\left(0.2: 0.2\right.$ of $\left.\mathrm{Fe}^{\mathrm{III}} / \mathrm{Fe}^{\mathrm{II}}\right)$ resulted in a respectable 59\% yield (entry 12). Extending the reaction time to compensate for the expected loss of reactivity from using less catalyst led to a lower rather than higher yield. Once the optimum reaction conditions were established (Table 1, entries 7 and 11), we began to explore the scope of the reaction (Scheme 1).

Modifying the alkene component revealed that the reaction worked with a wide range of functional groups (2a-k) (Scheme 1), although slight modifications of the reaction time and equivalents were required for more optimal results (see Scheme 1 footnotes). For example, the very low yield of $\mathbf{3 g}$ under the optimized conditions shown in Table 1 was greatly improved by increasing the reaction time to $48 \mathrm{~h}$. On the other hand, the presence of a Lewis basic substituent on the alkenes, as in the synthesis of $\mathbf{3 c}$ and $\mathbf{3 e} \mathbf{e}^{16}$ generally accelerated the reaction, which was usually completed within $8 \mathrm{~h}$. As might be expected, more substituted alkenes fared worse, the increased stability of the carboncentered radical favouring the reverse process. 


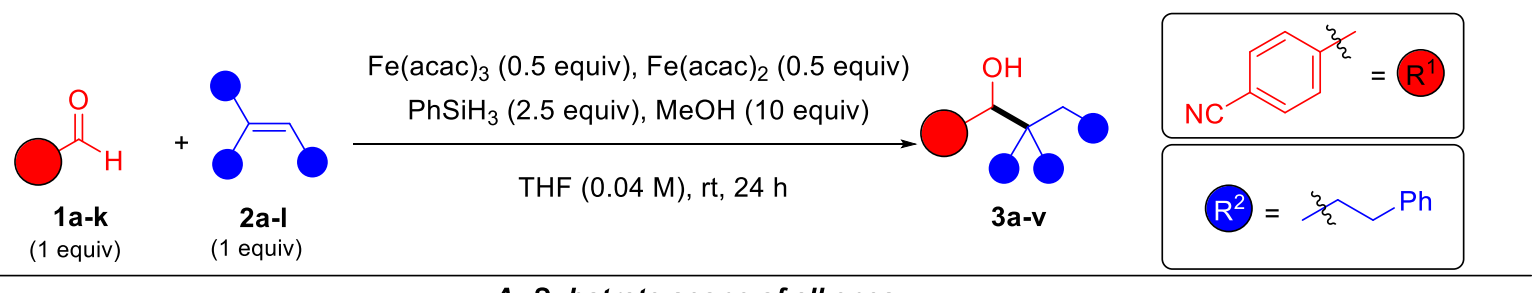

\section{A: Substrate scope of alkenes}

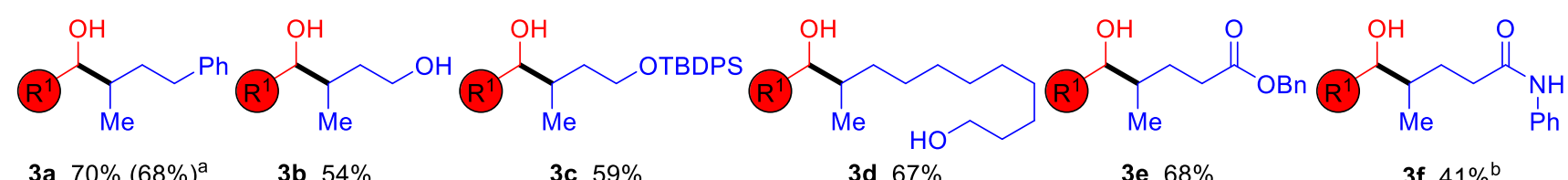

3a, $70 \%(68 \%)^{a}$

3b, $54 \%$

$3 c, 59 \%$

3d, $67 \%$

3e, $68 \%$

3f, $41 \%^{b}$

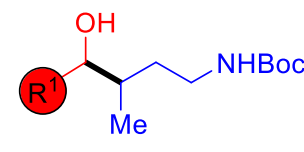

$3 g, 63 \%^{b}$

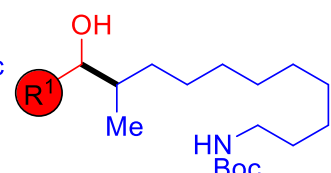

3h, $45 \%$

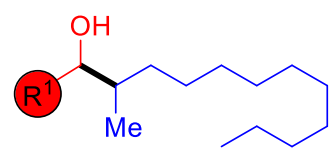

$3 \mathbf{i}, 47 \%$

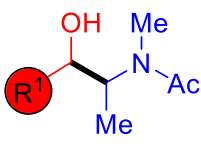

$3 j, 63 \%^{c}$

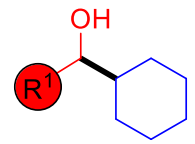

$3 k, 40 \%^{d}$

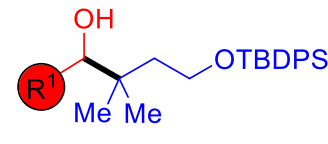

3I, $13 \%$

B: Substrate scope of carbonyl component<smiles>[R6]C(C)C(O)c1ccc(C(F)(F)F)cc1</smiles>

$3 \mathrm{~m}, 55 \%$<smiles>[R]C(C)C(O)c1ccccc1OC</smiles>

$3 \mathrm{r}, 81 \%^{\mathrm{b}, \mathrm{c}}$

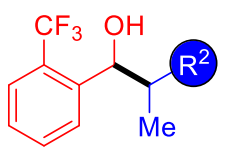

$3 n, 47 \%(40 \%)^{\mathrm{e}}$

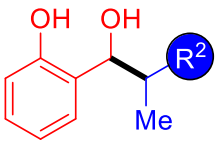

3s, $65 \%^{\mathrm{b}}$<smiles>[13CH]C([18OH])[C@H](O)c1ccc(F)cc1</smiles>

3o, $48 \%^{\text {b,d }}$<smiles>CC(C)C(O)c1ccsc1</smiles>

3t, $45 \%$<smiles>[13CH3]C([18OH])C(O)c1ccccc1</smiles>

$3 p, 42 \%$<smiles>[R6]C(C)C(O)c1ccccn1</smiles>

$3 u, 32 \%$<smiles>[R]C(C)C(O)c1ccc(OC)cc1</smiles>

$3 q, 52 \%^{\text {b,c }}$<smiles>[R6]C(C)C(O)CCc1ccccc1</smiles>

$3 v, 50 \%$

${ }^{a} 3.8 \mathrm{mmol}$ scale. ${ }^{b} 48 \mathrm{~h}$ instead of $24 \mathrm{~h} .{ }^{c} 2$ equivalents of alkene used. ${ }^{d} 2$ equivalents of aldehyde used. ${ }^{e} 2.9 \mathrm{mmol}$ scale.

Compound 3k needed extensive reaction optimisation to achieve a relatively moderate $40 \%$ yield, whilst 31 (derived from the tertiary radical intermediate) gave a disappointingly low $13 \%$ yield.

Variations in the acceptor showed that aromatic aldehydes (3m-s) with both electron-donating and withdrawing substituents are well tolerated. The results were strikingly improved when the benzaldehyde counterpart incorporated an oxygenated ortho substituent ( $\mathrm{OH}$ or $\mathrm{OMe}$ ) that can act as a Lewis base in the reactions leading to compounds 3r and 3s. Aromatic heterocycles were also feasible, such as thiophenes (3t) or pyridines (3u), as were aliphatic aldehydes (3v).

Given the important role of $\mathrm{Fe}^{\mathrm{II}}$ in the intermolecular coupling reaction, we next sought to evaluate its effects on the intramolecular version with ketones. ${ }^{5}$ One of the drawbacks of our previously reported method is that the reaction often required the use of stoichiometric Fe ${ }^{\text {III }}$ to be viable. Two examples were chosen to evaluate the utility of $\mathrm{Fe}^{\mathrm{II}}$ addition (Scheme 2) and after extensive screening it was found that the intramolecular reaction could also be greatly improved by the addition of $\mathrm{Fe}^{\mathrm{II}}$.
Scheme 2. Intramolecular couplings using $\mathrm{Fe}^{\mathrm{II}}$

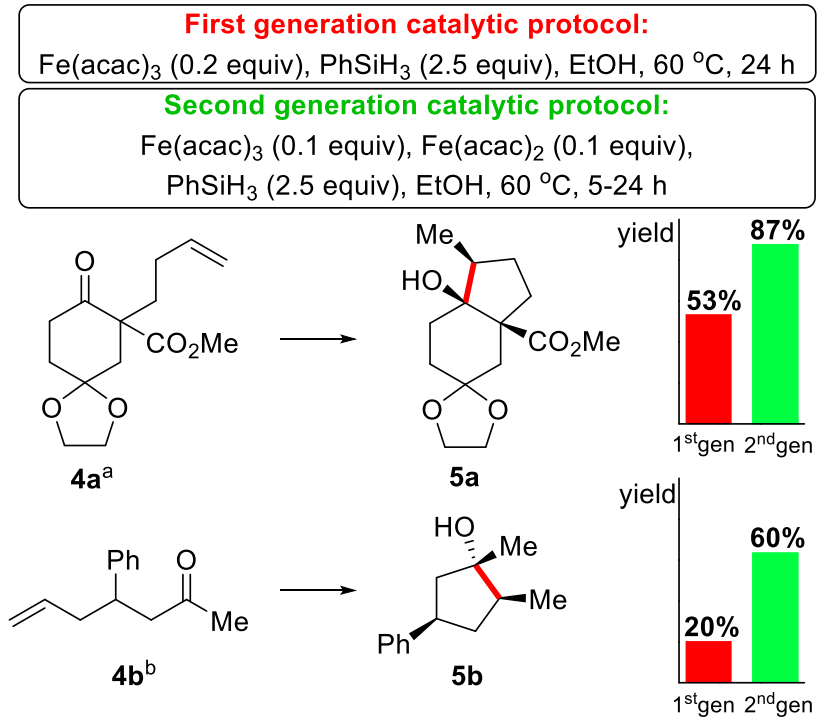

${ }^{a} \mathrm{PhSiH}_{3}$ was added for $4 \mathrm{~h}$ with a syringe pump and left to react for an additional $1 \mathrm{~h}$. ${ }^{b} \mathrm{All}$ components were reacted for $24 \mathrm{~h}$. 
It was possible to carry out the reactions with catalytic amounts of iron, although with some modifications. In contrast with the intermolecular version, ethanol was found to be an optimal solvent. Moreover, heating gave better outcomes, as did the controlled addition of phenylsilane via a syringe pump, which was key to minimizing the competing alkene reduction reaction in $\mathbf{4 a}$. For keto alkene $\mathbf{4 b}$, the reaction was slower and syringe pump addition of the silane afforded no beneficial effect; however, the addition of $\mathrm{Fe}^{\mathrm{II}}$ had a more notable impact, significantly improving the catalytic reaction yield from $20 \%$ to $60 \%$.

In summary, we have developed an intermolecular reductive $\mathrm{C}-\mathrm{C}$ coupling reaction of non-activated alkenes with aldehydes under MHAT conditions using both $\mathrm{Fe}^{\mathrm{II}}$ and $\mathrm{Fe}^{\mathrm{III}}$ salts. The use of carbonyls as intermolecular radical acceptors has long been hampered by reaction reversibility caused by the thermodynamically unstable alkoxyl radical intermediate. The good results obtained here hinged on the use of $\mathrm{Fe}^{\mathrm{II}}$, which is believed to play multiple beneficial synergistic roles in the reaction mechanism. Its application also allowed us to improve the conditions of our previous intramolecular version of the reaction, which often required stoichiometric iron to be effective. We hope that the ability to couple together two of the most common functional groups in synthetic chemistry (alkenes and aldehydes) in stoichiometric ratios, using cheap non-toxic reagents under operationally straightforward conditions, will prove useful for a great many applications. Mechanistic studies to elucidate the different functions of $\mathrm{Fe}^{\mathrm{II}}$ in the reaction are underway and will be reported in due course.

\section{ASSOCIATED CONTENT}

\section{Supporting Information}

The Supporting Information is available free of charge on the ACS Publications website at DOI:

Experimental procedures, characterization data, and NMR spectra (PDF)

FAIR Data is available as Supporting Information for Publication and includes the primary NMR FID files for compounds $3 \mathrm{a}-3 \mathrm{v}$.

\section{AUTHOR INFORMATION}

Corresponding Authors

Josep Bonjoch - Laboratori de Química Orgànica, Facultat de Farmàcia, IBUB, Universitat de Barcelona, 08028-Spain; orcid.org/0000-0002-5551-6720; Email: josep.bonjoch@ub.edu

Ben Bradshaw Laboratori de Química Orgànica, Facultat de Farmàcia, IBUB, Universitat de Barcelona, 08028-Spain; orcid.org/0000-0001-9612-9199; Email: benbradshaw@ub.edu

\section{Authors}

Mar Saladrigas Laboratori de Química Orgànica, Facultat de Farmàcia, IBUB, Universitat de Barcelona, 08028-Spain; orcid.org/0000-0001-7234-3705
Jordi Puig Laboratori de Química Orgànica, Facultat de Farmàcia, IBUB, Universitat de Barcelona, 08028-Spain; orcid.org/0000-0002-0363-8339

Complete contact information is available at:

https://pubs.acs.org/ /acs.orlett.

Notes

The authors declare no competing financial interest.

\section{ACKNOWLEDGMENTS}

Financial support for this research was provided by Project CTQ2016-75350-P (MINECO, Spain) / FEDER funds and PID2019-104188GB-I00 (MICINN, Spain). B.B. acknowledges the Serra Hunter program (Generalitat de Catalunya).

\section{REFERENCES}

(1) (a) Gray, P.; Williams, A. The Thermochemistry and Reactivity of Alkoxyl Radicals. Chem. Rev. 1959, 59, 239-328. (b) Hioe, J.; Zipse, H. Radical stability and its role in synthesis and catalysis. Org. Biomol. Chem. 2010, 8, 3609-3617. (2) (a) Beckwith, A. L. J.; Hay, B. P. Kinetics of the Reversible $\beta$ Scission of the Cyclopentyloxy Radical. J. Am. Chem. Soc. 1989, 111, 230-234. (b) Beckwith, A. L. J.; Hay, B. P. Kinetics and Mechanism of the Exo Cyclizations of $\omega$-Formylalkyl Radicals. J. Am. Chem. Soc. 1989, 111, 2674-2681. (c) Dowd, P.; Zhang, W. Free radical-mediated ring expansion and related annulations. Chem. Rev. 1993, 93, 2091-2115. (d) Wilsey, S.; Dowd, P.; Houk, K. N. Effect of Alkyl Substituents and Ring Size on Alkoxy Radical Cleavage Reactions. J. Org. Chem. 1999, 64, 8801-8811. (3) (a) Nishikawa, K.; Ando, T.; Maeda, K.; Morita, T.; Yoshimi, Y. Photoinduced Electron Transfer Promoted Radical Ring Expansion and Cyclization Reactions of $\alpha-(\omega$-Carboxyalkyl $)$ $\beta$-Keto Esters. Org. Lett. 2013, 15, 636-638, and references therein. (b) Ota, E.; Wang, H.; Frye, N. L.; Knowles. R. R. A Redox Strategy for Light-Driven, Out-of-Equilibrium Isomerizations and Application to Catalytic C-C Bond Cleavage Reactions. J. Am. Chem. Soc. 2019, 141, 1457-1462, and references therein. (4) (a) Nishida, A.; Takahashi, H.; Takeda, H.; Takada, N.; Yonemitsu, O. Sequential Radical Cyclization, Alkoxy Radical Fragmentation, and Recyclization Processes: A Novel Method for the Synthesis of Fused Cycloheptanones and Cyclooctenones from Cyclohexanones. J. Am. Chem. Soc. 1990, 112, 902-904. (b) Fernández-Mateos, A.; Martín De La Nava, E.; Pascual Coca, G.; Ramos Silvo, A.; Rubio González, R. Radicals from Epoxides. Intramolecular Addition to Aldehyde and Ketone Carbonyls. Org. Lett. 1999, 1, 607-609. (c) Devin, P.; Fensterbank, L.; Malacria, M. Tin-Free Radical Chemistry: Intramolecular Addition of Alkyl Radicals to Aldehydes and Ketones. Tetrahedron Lett. 1999, 40, 5511-5514. (d) Zheng, H.-X.; Shan X.-H, Qu, J.-P.; Kang, Y.-B. Strategy for Overcoming Full Reversibility of Intermolecular Radical Addition to Aldehydes: Tandem $\mathrm{C}-\mathrm{H}$ and $\mathrm{C}-\mathrm{O}$ Bonds Cleaving Cyclization of (Phenoxymethyl)arenes with Carbonyls to Benzofurans. Org. Lett. 2018, 20, 3310-3313. (e) Xie, S.; Li, D.; Huang, H.; Zhang, F.; Chen. Y. Intermolecular Radical Addition to Ketoacids Enabled by Boron Activation. J. Am. Chem. Soc. 2019, 141, 16237-16242. (f) Shi, S.; Kuo, J. L.; Chen, T.; Norton, J. R. Catalytic Cycloisomerization onto a Carbonyl Oxygen. Org. 
Lett. 2020, 22, 6171-6176.

(5) Saladrigas, M.; Bosch, C.; Saborit, G. V.; Bonjoch, J.; Bradshaw, B. Radical Cyclization of Alkene-Tethered Ketones Initiated by Hydrogen-Atom Transfer. Angew. Chem. Int. Ed. 2018, $57,182-186$.

(6) For reviews on MHAT, see: (a) Crossley, S. W. M.; Martinez, R. M.; Obradors, C.; Shenvi, R. A. Mn, Fe, and Co-Catalyzed Radical Hydrofunctionalizations of Olefins. Chem. Rev. 2016, 116, 8912-9000. (b) Green, S. A.; Crossley, S. W. M.; Matos, J. L. M.; Vásquez-Céspedes, S.; Shevick, S. L.; Shenvi, R. A. The High Chemofidelity of Metal-Catalyzed Atom Transfer. Acc. Chem. Res. 2018, 51, 2628-2640.

(7) (a) Gualandi, A.; Mengozzi, L.; Cozzi, P. G. Iron-Promoted Radical Reactions: Current Status and Perspectives. Asian J. Org. Chem. 2017, 6, 1160-1179. (b) Lübken, D.; Saxarra, M.; Kalesse, M. Tris(acetylacetonato) Iron(III): Recent Developments and Synthetic Applications. Synthesis, 2019, 51, 161177.

(8) (a) Pitzer, L.; Sandfort F.; Strieth-Kalthoff, F.; Glorius, F. Intermolecular Radical Addition to Carbonyls Enabled by Visible Light Photoredox Initiated Hole Catalysis. J. Am. Chem. Soc. 2017, 139, 13652-13655. For another example using photocatalysis see: (b) Kawamoto, T.; Fukuyama, T.; Ryu, I. Radical Addition of Alkyl Halides to Formaldehyde in the Presence of Cyanoborohydride as a Radical Mediator. A New Protocol for Hydroxymethylation Reaction. J. Am. Chem. Soc. 2012, 134, 875877.

(9) (a) Matos, J. L. M.; Vásquez-C., S.; Gu, J.; Oguma, T.; Shenvi, R. A. Branch-Selective Addition of Unactivated Olefins into Imines and Aldehydes. J. Am. Chem. Soc. 2018, 140, 16976-16981. For a review of this approach see (b) Pitzer, L.; Schwarz, J, L.; Glorius, F. Reductive radical-polar crossover: traditional electrophiles in modern radical reactions. Chem. Sci., 2019, 10, 82858291. For other related examples of this approach see: (c) Nishikawa, K.; Ando, T.; Maeda, K.; Morita, T.; Yoshimi, Y. Photoinduced Electron Transfer Promoted Radical Ring Expansion and Cyclization Reactions of $\alpha$ - $(\omega$-Carboxyalkyl $) \beta$-Keto Esters. Org. Lett. 2013, 15, 636-638. (d) Schwarz, J. L.; Schäfers, F.; Tlahuext-Aca, A.; Lückemeier, L.; Glorius, F. Diastereoselective Allylation of Aldehydes by Dual Photoredox and Chromium Catalysis. J. Am. Chem. Soc. 2018, 140, 12705-12709. (e) Mitsunuma, H.; Tanabe, S.; Fuse, H.; Ohkubo, K.; Kanai, M. Catalytic asymmetric allylation of aldehydes with alkenes through allylic C(sp3)-H functionalization mediated by organophotoredox and chiral chromium hybrid catalysis. Chem. Sci., 2019, 10, 3459-3465. (f) Gualandi, A.; Calogero, F.; Mazzarini, M.; Guazzi, S.; Fermi, A.; Bergamini, G.; Cozzi, P. G. $\mathrm{Cp}_{2} \mathrm{TiCl}_{2}$-Catalyzed Photoredox Allylation of Aldehydes with Visible Light. ACS Catal. 2020, 10, 3857-3863.

(10) Saladrigas, M.; Loren, G.; Bonjoch, J.; Bradshaw, B. Hydrogen Atom Transfer (HAT)-Triggered Iron-Catalyzed Intra- and Intermolecular Coupling of Alkenes with Hydrazones: Access to Complex Amines. ACS Catal. 2018, 8, 11699-11703. (11) Saladrigas, M.; Bonjoch, J.; Bradshaw, B. Iron Hydride Radical Reductive Alkylation of Unactivated Alkenes. Org. Lett. 2020, 22, 684-688.

(12) (a) Lo, J. C.; Kim, D.; Pan, C.-M.; Edwards, J. T.; Yabe, Y.; Gui, J.; Qin, T.; Gutierrez, S.; Giacoboni, J.; Smith, M. W.; Holland, P. L.; Baran, P. S. Fe-Catalyzed C-C Bond Construction from Olefins and Radicals. J. Am. Chem. Soc. 2017, 139, 2484-2503. (b) Kim, D.; Rahaman, S. M. W.; Mercado, B. Q.; Poli, R.; Holland, P. L. Roles of Iron Complexes in Catalytic Radical Alkene CrossCoupling: A Computational and Mechanistic Study. J. Am. Chem.
Soc. 2019, 141, 7473-7485.

(13) Leifert, D.; Studer, A. The Persistent Radical Effect in Organic Synthesis. Angew. Chem. Int. Ed. 2020, 59, 74-108. (14) Dao, H. T.; Li, C.; Michaudel, Q.; Maxwell, B. D.; Baran, P. S. Hydromethylation of Unactivated Olefins. J. Am. Chem. Soc. 2015, 137, 8046-8049.

(15) Mukaiyama, T.; Yamada, T. Recent Advances in Aerobic Oxygenation. Bull. Chem. Soc. Jpn. 1995, 68, 17-35. (16) It is noteworthy that $\delta$-hydroxy ester $\mathbf{3 e}$ (mixture of epimers) underwent a partial lactonization process both under chromatography $\left(\mathrm{SiO}_{2}\right)$ and on standing in $\mathrm{CDCl}_{3}$ (see Supporting Information). Moreover, some lactone formation in the reaction medium cannot be ruled out. 